\title{
Relaciones entre calcio, ácidos grasos no esterificados, e insulina sanguínea en preparto y leche bovina perdida en el inicio de la lactancia Calcium, non-esterified fatty acids, and insulin in blood in prepartum and their relationships with milk yield losses at early lactation
}

Rufino López Ordaz ${ }^{1}$, Tomás Tinajero Pérez ${ }^{1}$, Reyes López Ordaz ${ }^{2}$, Germán David Mendoza Martínez ${ }^{2}$, Juan de Dios Roldán Montiel ${ }^{3}$, Adelfo Vite Aranda ${ }^{3}$ y Agustín Ruíz Flores ${ }^{1}$

Palabras Clave: ácidos grasos no esterificados; insulina; calcio; preparto; postparto

\section{Recepción: 07-07-17 / Aceptación: 20-09-17}

\section{Resumen}

\section{Introducción}

El objetivo fue determinar la relación entre las concentraciones séricas de calcio $\left(\mathrm{Ca}^{2+}\right)$, ácidos grasos no esterificados (AGNE) e insulina preparto con pérdidas en la producción de leche (PL) en el inicio de la lactancia.

\section{Método}

Doscientas cuarenta y tres vacas Holstein-Friesian (780 \pm 36 kg PV; con más de dos lactancias) fueron muestreadas por punción coccígea varias veces antes, durante y después del parto. Para cada muestreo y cada metabolito, las concentraciones séricas se estratificaron con umbrales y se relacionaron con la PL.

\section{Resultados}

Cuando los niveles de $\mathrm{Ca}^{2+}$ fueron bajos 10 días antes del parto y se relacionaron con la PL a los 10 días posparto, se observó que el $77.11 \%$ de las vacas perdieron $2.20 \mathrm{~kg}$ de leche; mientras en el día 20, el $66.41 \%$ de las vacas perdieron $1.40 \mathrm{~kg}$ animal $^{-1}$ día $^{-1}$. Por el contrario, cuando los AGNE fueron altos 10 días antes de parto y se relacionaron con PL, $67.40 \%$ de las vacas mostraron riesgo de perder $0.80 \mathrm{~kg}$, mientras que a los 20 días posparto, el $22.00 \%$ de las vacas perdieron aproximadamente $0.90 \mathrm{~kg} \mathrm{vaca}^{-1}$ día $^{-1}$. La determinación de insulina preparto baja y PL en los días 10 y 20 de lactancia indicó que $6.02 \%$ de las vacas perdieron 0.80 y $0.70 \mathrm{~kg}$ de leche animal ${ }^{-1}$ día $^{-}$

${ }^{1}$ para los días 10 y 20 de lactancia.

\footnotetext{
${ }^{1}$ Departamento de Zootecnia, Universidad Autónoma Chapingo. E-mail: aruizf1959@gmail.com

${ }^{2}$ Departamento de Producción Agrícola y Animal, Universidad autónoma Metropolitana. Unidad Xochimilco.

${ }^{3}$ Grupo Biotecap, S. A. de C. V.

(C) Universidad De La Salle Bajío (México)
} 


\section{Conclusión}

En conclusión, niveles bajos de calcio, AGNE alto e insulina baja en sangre, obtenidos 10 días antes de parto fueron asociados con pérdidas en producción de leche en los días 10 y 20 después del parto. También se observó que los niveles bajos de calcio podrían causar mayores pérdidas de leche que los niveles altos de ácidos grasos no esterificados y las concentraciones bajas de insulina preparto.

\section{Abstract \\ Introduction}

The objective was to determine the relationship between the serum concentrations of Calcium $\left(\mathrm{Ca}^{2+}\right)$, non-esterified fatty acids (NEFA), and insulin 10 days prepartum with losses in milk yield (MY) at 10 and 20 days of lactation.

\section{Method}

Two hundred forty-three Holstein-Friesian cows (780 $\pm 36 \mathrm{~kg} \mathrm{PV}$, with more than two lactations) were sampled by coccygeal puncture several times before, during and after parturition.

\section{Results}

When $\mathrm{Ca}^{2+}$ levels were low 10 days before parturition and were related to MY at 10 days postpartum, it was observed that $77.11 \%$ of the cows lost $2.20 \mathrm{~kg}$ of milk; while on day $20,66.41 \%$ of the cows lost $1.40 \mathrm{~kg} \mathrm{animal}^{-1}$ day $^{-1}$. In contrast, when NEFA were high 10 days before calving and were related to MY, $67.40 \%$ of cows showed a risk of losing $0.80 \mathrm{~kg}$, whereas at 20 days postpartum, $22.00 \%$ of cows lost approximately $0.90 \mathrm{~kg} \mathrm{cow}^{-1} \mathrm{day}^{-1}$. The determination of low prepartum insulin and MY on days 10 and 20 of lactation indicated that $6.02 \%$ of cows lost 0.80 and $0.70 \mathrm{~kg}_{\text {of }}$ milk $^{-1}$ animal $^{-1} \mathrm{day}^{-1}$ for days 10 and 20 of lactation.

\section{Conclusion}

In conclusion, low blood levels of calcium, high levels of NEFA, and low insulin 10 days before parturition were associated with milk yield losses at 10 and 20 days after parturition. 
It was also observed that low calcium could cause greater milk losses than excesses of nonesterified fatty acids and serum low insulin concentrations.

Keywords: non-esterified fatty acid; calcium; insulin; prepartum; postpartum

\section{Introducción}

El mantenimiento de la salud de los animales y el incremento en la productividad del hato son los retos más difíciles que enfrenta el productor lechero. El periodo alrededor del parto es crítico debido a la reducción en el consumo de materia seca (CMS), incrementos en la demanda de nutrientes, energía y calcio $\left(\mathrm{Ca}^{2+}\right)$ para el mantenimiento y la síntesis de leche. Debido a la deficiencia en el consumo, los requerimientos no pueden cubrirse y el déficit permite que el animal entre en balance energético negativo (BEN).

Durante el BEN al inicio de la lactancia, se ha observado que la concentración de glucosa en suero sanguíneo es baja, y paralelamente incrementa la concentración de ácidos grasos no esterificados (AGNE; Gross et al. 2011), y los cuerpos cetónicos (Gross et al. 2011). El cuerpo cetónico circulante más sobresaliente en rumiantes es ß-hydroxibutitrato ( $(\mathrm{HB})$, que puede usarse como fuente de energía en tejidos corporales como cerebro y corazón (Veech, 2004), riñón y músculo esquelético (Riderman y Goodman, 1973), y la glándula mamaria de las vacas lactantes. Sin embargo, el incremento en la concentración de $\beta \mathrm{HB}$ por arriba de $1.2 \mathrm{mmol} \mathrm{L}^{-1}$ es un indicador de cetosis subclínica en vacas lecheras, lo que tiene efectos detrimentales en la salud y comportamiento (Ospina et al. 2010). El incremento de $\beta \mathrm{HB}$ en plasma reduce la glucosa circulante en ovejas (Schlumbohn y Harmeyer, 2004) e incrementa el riesgo de cetosis, desplazamiento de abomaso y metritis con la consecuente reducción de la producción en vacas lecheras (Duffield et al. 2009). También puede ocurrir que se presente hipocalcemia en los primeros días después del parto (Reinhardt et al. 2011).

Las medidas de las concentraciones de $\mathrm{Ca}^{2+}$, AGNE, e insulina antes y después del parto pueden ser indicadores potenciales de la habilidad de la vaca para cubrir los retos metabólicos en el periodo de transición, y posiblemente, permitir predecir algunos riesgos de enfermedades y la posible reducción de leche en el inicio de la lactancia. Las concentraciones de $\mathrm{Ca}^{2+}$ demuestran la capacidad de la vaca para reemplazar la pérdida de $\mathrm{Ca}^{2+}$ extracelular en el proceso de producción 
de leche y el balance entre el hueso, y la eficiencia de absorción de insulina y $\mathrm{Ca}^{2+}$ (Horst et al. 1994). Las concentraciones séricas de AGNE sirven como indicador de la movilización de la grasa corporal y reflejan la particularidad de la vaca para adaptarse al BEN; mientras que la insulina puede servir como indicador del metabolismo de carbohidratos.

A nivel de la vaca, las reducciones en las concentraciones séricas de $\mathrm{Ca}^{2+}$, los incrementos en AGNE y las reducciones en insulina se han asociado con el incremento en el riesgo de contraer enfermedades (Seifi et al. 2011) y pérdida de leche (Chapinal et al. 2012a). Los umbrales a nivel de vacas de estos metabolitos se han utilizado para identificar individuos con riesgos de dañar la salud y la productividad de la misma. Sin embargo, las intervenciones individuales para minimizar los efectos indeseables del BEN e hipocalcemia, cetosis u otros trastornos metabólicos alrededor del parto son difíciles de lograr. Con base en lo anterior, el objetivo del presente estudio fue determinar las concentraciones séricas de $\mathrm{Ca}^{2+}$, ácidos grasos no esterificados, e insulina a los diez días antes del parto, y relacionarlas con las pérdidas de leche a los 10 y 20 días de lactancia en vacas Holstein-Friesian en confinamiento.

\section{Método}

\section{Localización del área de estudio}

El estudio se llevó a cabo en tres establos lecheros de la Comarca Lagunera, ubicados en un radio no mayor de 150 kilómetros. Los establos se seleccionaron con base en la disponibilidad de sus encargados para participar en el estudio; los elegidos cumplieron con los criterios de tener aproximadamente 2,000 vacas en línea de ordeño, dos ordeños por día y dietas completas totalmente mezcladas de sorgo-soya (Cuadro 1). El establo uno se localiza en San Pedro, Coahuila, a 1,100 m (25 ${ }^{\circ} 44^{\prime} 36^{\prime \prime} \mathrm{N}$ y $103^{\circ} 10^{\prime} 22^{\prime}$ O). El establo dos se localiza en Francisco I. Madero, Coahuila, a $1,108 \mathrm{~m}\left(25^{\circ} 48^{\prime} 25^{\prime}\right.$ ” N y $103^{\circ} 13^{\prime} 32^{\prime}$ O $)$, y el tres, se localiza en Torreón, Coahuila, a 1,120 m (25 '51’86” N y 103 `34’36” O). El clima de la región es árido y la precipitación es aproximadamente $300 \mathrm{~mm}$ anuales distribuidos principalmente de julio a septiembre (García, 2005). 


\section{Manejo y medición de las vacas}

Los animales utilizados en el estudio fueron Holstein-Friesian, de aproximadamente 40 días antes de la fecha probable de parto. El peso corporal (PC) aproximado fue $780 \pm 36 \mathrm{~kg}$, con condición corporal (CC) de 3.0 (escala 1, delgada; 5, gorda; Wildam et al. 1982) y más de dos lactancias. Con base en reportes previos, aproximadamente 30 vacas por hato fueron suficientes para detectar la prevalencia de concentraciones elevadas de metabolitos en un intervalo de confianza de 5 a 95 \% (Análisis de umbrales, intervalos de Confianza de Wald). Las fechas probables de partos se obtuvieron de las listas generadas por el software Dairy Comp 305 (Dairy Comp 305, Valley Ag Software, Tulare, CA; PC Dart, Dairy Records, Management System, Raleigh, NC).

Los animales se seleccionaron por PL en la lactancia previa, CC y aparentemente libres de enfermedades. Los muestreos incluyeron -30, -20, -10, -3, -2 y -1 día antes del parto, el día del parto, y 1, 2, 3, 10, 20 y 30 días postparto. En cada muestreo, se colectó una muestra de sangre de la vena coccígea, de aproximadamente $10.0 \mathrm{~mL}$, en tubos vacutainer sin anticoagulante (Beckton-

Dickinson, Franklin Lakes, NJ). Las muestras se dejaron a temperatura ambiente para su coagulación por una hora. La sangre se centrifugó a 1000 x g durante 25 minutos a $4{ }^{\circ} \mathrm{C}$, el suero se separó y se almacenó a $-20^{\circ} \mathrm{C}$ hasta su uso. La evaluación de la CC se realizó cada semana; y se evaluó con una escala de 1: delgada y 5: gorda con variaciones de 0.25 . Los encargados de los establos fueron instruidos para observar enfermedades o eventos no saludables en los animales. Dichos eventos incluyeron desplazamiento de abomaso, partos distócicos, cetosis, mastitis, metritis, fiebre de leche y retención placentaria. Los técnicos instruyeron a los encargados de establos mediante discusiones verbales o boletines con las definiciones de las enfermedades, para uniformizar criterios y estandarizar la información colectada. Las definiciones de las enfermedades fueron descritas previamente por LeBlanc et al. (2010).

\section{Análisis del suero sanguíneo}

Las muestras se analizaron para $\mathrm{Ca}^{2+}$, AGNE e insulina en los laboratorios de la Facultad de Medicina Veterinaria y Zootecnia de la Universidad Nacional Autónoma de México (UNAM). El $\mathrm{Ca}^{2+}$ se analizó con un kit para $\mathrm{Ca}^{2+}$ denominado Calcium Colorimetric Method; distribuido por Randox Laboratories Limited (County Antrim, UK). Los AGNE se determinaron con un kit HR series NEFA-HR [(2 ACS (Acyl-CoA sintasa)-ACOD (Acyl-CoA-oxidasa) method Wako Pure Chemical Industries, Mountain View, CA, USA)] siguiendo los procedimientos de los fabricantes. 
La determinación de insulina se realizó mediante ensayo por inmunoabsorción ligada a enzimas (ELISA).

\section{Análisis del alimento}

En el Cuadro 1 se presenta la composición química de las dietas experimentales. Las muestras de alimento correspondieron a dietas diferentes de acuerdo a las etapas y los establos. El día 30 preparto correspondió a dietas en transición; mientras que el día -10 preparto fue de reto y finalmente, a los +30 días postparto fue para vacas frescas. Las muestras se pesaron y secaron a $55-65^{\circ} \mathrm{C}$ en una estufa de aire forzado por $48 \mathrm{~h}$, y posteriormente, fueron nuevamente pesadas para determinar la materia seca de campo parcial. Subsiguientemente, las muestras se molieron en un molino Wiley (A. H. Thomas, Philadelphia, PA). La materia seca total se determinó utilizando una estufa a $100^{\circ} \mathrm{C}$ durante $24 \mathrm{~h}$. Para determinar el contenido de cenizas y materia orgánica (MO) las muestras se incineraron en mufla a $500^{\circ} \mathrm{C}$, por $12 \mathrm{~h}$. Las fibras detergente neutro (FDN) y detergente ácido (FDA) se determinaron usando el método de Goering y Van Soest (1970). El contenido de proteína cruda (PC) se determinó con el método Kjeldahl (AOAC, 2006).

\section{Análisis estadísticos}

La información fue analizada en un paquete computacional Statistical Analysis System (SAS, 2014 versión 9.4); de acuerdo a los criterios siguientes: 1) Las diferencias entre AGNE, $\mathrm{Ca}^{2+}$ e insulina en vacas, las diferencias entre ranchos y fechas de muestreos fueron analizados con el procedimiento mixed (SAS, 2014). Para ello, se utilizó un modelo mixto con medidas repetidas dentro de vaca (anidadas dentro de hato) y considerando hato como aleatorio. 2) La CC fue categorizada como delgada $(\leq 2.0)$, moderada $(2.25-3.00)$ o gorda $(\geq 4.0)$; mientras que la PL se agrupó como baja $(\leq 18.20)$, media $\left(18.21\right.$ - 36.33) y alta $\left(\geq 36.33 \mathrm{~kg} \mathrm{~d}^{-1}\right)$. Para determinar la asociación entre PL y las concentraciones séricas de $\mathrm{Ca}^{2+}, \mathrm{AGNE}$, e insulina se realizaron análisis univariados por fecha de muestreo con variables categóricas como fecha de parto, CC y establo.

Para cada metabolito u hormona y fecha de muestreo, se hicieron al menos tres tipos de umbrales (bajo, medio y alto). Los umbrales se formaron siguiendo la metodología propuesta por Chapinal et al. (2011). Para predecir el volumen de leche perdida o no cosechada, se utilizó la categorización de las vacas en grupos de bajo, medio y alto riesgo. Para los días 10 preparto, 10 y 20 posparto los 
umbrales considerados a nivel de vaca fueron: $\mathrm{Ca}^{2+} \leq 2.1 \mathrm{mmol} \mathrm{L}^{-1}$, AGNE $\geq 0.5 \mathrm{mmol} \mathrm{L}^{-1}$, e insulina $\leq 0.26 \mathrm{ng} \mathrm{mL}^{-1}$.

En cada fecha de muestreo y en cada umbral se calculó la proporción de grupos de animales muestreados de riesgo alto para cada establo. En forma similar, para cada fecha de muestreo y para cada umbral, se crearon grupos con incrementos de 5.0 puntos porcentuales de la proporción de animales en el grupo de riesgo alto (p. ej. $\geq 10 \%$ de vacas con AGNE preparto $\geq 0.5 \mathrm{mmol} \mathrm{L}^{-1}, \geq$ $15 \%$ con $\geq 0.5 \mathrm{mmol} \mathrm{L}^{-1}, \mathrm{y} \geq 20 \%$ con $\geq 0.5 \mathrm{mmol} \mathrm{L}^{-1}$, y así sucesivamente).

\begin{tabular}{|l|l|l|l|l|l||l|l|l|}
\hline $\begin{array}{c}\text { Dieta } \\
\text { ofrecidas, días }\end{array}$ & Establo & $\begin{array}{c}\text { Cenizas, } \\
\mathbf{\%}\end{array}$ & $\begin{array}{c}\text { FDN, } \\
\mathbf{\%}^{\mathbf{a}}\end{array}$ & $\begin{array}{c}\text { FDA, } \\
\mathbf{\%}^{\mathbf{b}}\end{array}$ & $\begin{array}{c}\text { Proteína } \\
\mathbf{\%}\end{array}$ & $\begin{array}{c}\mathbf{E N}_{\mathbf{L}}^{\mathbf{c}}, \\
\mathbf{M c a l}_{\mathbf{~ k g}}^{-} \\
\mathbf{1}\end{array}$ & $\begin{array}{c}\text { EE, \% } \\
\text { ( }\end{array}$ & $\begin{array}{c}\text { Fibra } \\
\text { cruda, } \\
\mathbf{\%}\end{array}$ \\
\hline$-30,-20$ & 1 & 15.10 & 46.20 & 31.02 & 13.10 & 1.46 & 3.00 & 23.11 \\
\hline$-30,-20$ & 2 & 8.30 & 33.00 & 13.00 & 13.40 & 1.42 & 2.20 & 8.30 \\
\hline$-30,-20$ & 3 & 12.00 & 55.10 & 29.00 & 13.77 & 1.41 & 2.10 & 13.20 \\
\hline-10 & 1 & 11.05 & 44.00 & 26.10 & 14.01 & 1.46 & 4.00 & 24.01 \\
\hline-10 & 2 & 9.00 & 43.01 & 21.00 & 14.20 & 1.42 & 4.10 & 12.11 \\
\hline-10 & 3 & 12.10 & 47.03 & 26.00 & 13.88 & 1.41 & 2.11 & 13.02 \\
\hline$+10,+20,+30$ & 1 & 10.10 & 37.00 & 19.20 & 16.33 & 1.81 & 5.11 & 18.12 \\
\hline$+10,+20,+30$ & 2 & 10.00 & 40.00 & 21.00 & 18.00 & 1.79 & 4.01 & 13.11 \\
\hline$+10,+20,+30$ & 3 & 9.33 & 41.02 & 18.00 & 17.11 & 1.80 & 5.01 & 12.00 \\
\hline
\end{tabular}

Cuadro 1. Composición química de las dietas experimentales ofrecidas a vacas Holstein-Friesian en confinamiento antes (-30, -20 y -10 días), y después del parto $(+10,+20$ y +30 días $)$ en tres establos comerciales. ${ }^{a}$ Fibra detergente neutro; ${ }^{b}$ Fibra detergente neutro; ${ }^{c}$ Energía neta de lactancia; ${ }^{d}$ Extracto etéreo.

Para estudiar la diferencia entre umbrales se crearon variables Dummy jerárquicas designando el valor de 1.0 para las vacas consideradas de riesgo alto y 0.0 para riesgo bajo (por ejemplo, vacas con concentraciones de AGNE preparto $\geq 0.5 \mathrm{mmol} \mathrm{L}^{-1}$, se le asignó el valor de 1.0 para ese umbral particular y con concentraciones de AGNE preparto $<0.5 \mathrm{mmol} \mathrm{L}^{-1}$, se le asignó un valor de 0.0 ). En la mayoría de los umbrales, el nivel medio funcionó como el punto de referencia; 3) Las diferencias en los resultados entre las vacas de bajo y alto riesgo fueron contrastados usando modelos lineales generales (GLM; SAS, 2014). La proporción de animales muestreados y diagnosticados con niveles riesgosos se consideraron como variables continúas, y las vacas tomaron valores entre 0.0 y 1.0. Para asegurar que los estimadores e intervalos de confianza fueran 
calculados correctamente, se usó la transformación logit con un factor de corrección por sesgo de 0.25 a 0.50 y se aplicó a los animales muestreados. Esta transformación fue necesaria para cubrir las suposiciones de normalidad y homogeneidad de varianzas; y 4) Para cada umbral de concentraciones, se crearon modelos multivariados que se analizaron por regresión logística condicional (SAS, 2014) incluyendo las proporciones dicotomizadas de animales en el grupo de riesgo alto.

Cuando fue requerido, las variables e interacciones fueron removidas del modelo final manualmente si $p>0.05$. De cada modelo, el umbral con coeficiente $\beta$ positivo en la escala logit (caso de AGNE, $\mathrm{Ca}^{2+}$ e insulina preparto) y un coeficiente negativo (caso de PL), y los valores de probabilidad más bajos fueron elegidos como los umbrales óptimos para predecir pérdidas de PL en los días 10 y 20 postparto. En el caso de PL donde se aplicó la transformación logit, los coeficientes fueron retransformados. Los resultados se presentan como coeficientes de disparidad y los intervalos de confianza entre animales por arriba y por debajo del umbral de referencia. El coeficiente de disparidad expresa la ventaja o probabilidad de experimentar un evento $(e . g$. leche producida o no cosechada) para un grupo de riesgo alto (por arriba del umbral) cuando se compara con uno de riesgo bajo (por debajo del umbral). Los resultados de PL se presentan como la diferencia en PL $\left(\mathrm{kg} \mathrm{d}^{-1}\right) \pm$ el error estándar entre umbrales por arriba o abajo del umbral del hato.

\section{Resultados}

En total, 253 registros se utilizaron para establecer las relaciones entre las concentraciones séricas de $\mathrm{Ca}^{2+}$ AGNE, e insulina a -30, -20, -10, -3, -2, y -1 días preparto, el día de parto, y 1, 2, 3, 10, 20 y 30 días postparto con PL. El análisis de la composición química de las dietas utilizadas se muestra en el Cuadro 1. Durante el periodo seco, las vacas recibieron dietas con aproximadamente $13.00 \%$ de proteína, y 1.42 Mcal de $\mathrm{EN}_{\mathrm{L} \mathrm{kg}}{ }^{-1}$; mientras que el postparto las dietas variaron de 16.00 a18.00 $\%$ de PC y 1.79 a 1.81 Mcal de $\mathrm{EN}_{\mathrm{L} \mathrm{kg}}{ }^{-1}$. La PL fue mayor ( $\mathrm{p}<0.0001$ ) en el establo tres en comparación con uno y dos. Por el contrario, la CC fue similar entre establos (Cuadro 2). La concentración de AGNE fue similar para los establos uno y tres; mientras que el establo dos tuvo concentraciones menores $(\mathrm{p}<0.01)$. Las vacas del establo uno tuvieron mayor concentración ( $\mathrm{p}$ $<0.001) \mathrm{de} \mathrm{Ca}^{2+}$ en comparación con dos y tres; mientras que insulina fue similar en todos los establos ( $\mathrm{p}>0.34)$. 
La Figura 1 muestra las concentraciones séricas de los metabolitos y hormona obtenidas por fecha de muestreo independientemente del establo de procedencia. En los primeros análisis, las concentraciones de $\mathrm{Ca}^{2+}$ bajos, AGNE bajos, e insulina intermedios, y en los primeros 10 días antes del parto se asociaron con volúmenes de leche perdidos o no cosechados en los días 10 y 20 postparto, cuando cada metabolito fue analizado por separado.

\begin{tabular}{|c|c|c|c|c|c|c|}
\hline \multirow[b]{2}{*}{ Ítem } & \multirow[b]{2}{*}{ Establo 1} & \multirow[b]{2}{*}{ Establo 2} & \multirow[b]{2}{*}{ Establo 3} & \multicolumn{3}{|c|}{$P$} \\
\hline & & & & Trt & $\begin{array}{l}\text { Tiem } \\
\text { po }(\mathbf{T})\end{array}$ & $\begin{array}{l}\text { Trt } \\
\times \text { T }\end{array}$ \\
\hline \multicolumn{7}{|l|}{$\begin{array}{l}\text { Producción de leche, } \\
\mathrm{kg} \mathrm{d}^{-1}\end{array}$} \\
\hline Día 10, lactancia & $25.9 \pm 3.92^{\mathrm{b}}$ & $\begin{array}{l}26.02 \\
3.65^{\mathrm{b}}\end{array}$ & $\begin{array}{l}33.75 \\
6.14^{\mathrm{a}}\end{array}$ & $\begin{array}{l}0.000 \\
1\end{array}$ & $\begin{array}{l}0.000 \\
1\end{array}$ & 0.99 \\
\hline Día 20, lactancia & $\begin{array}{l}27.03 \\
3.92^{\mathrm{b}}\end{array}$ & $\begin{array}{l}29.30 \\
2.90^{\mathrm{b}}\end{array}$ & $\begin{array}{l}32.46 \\
4.42^{\mathrm{b}}\end{array}$ & $\begin{array}{l}0.000 \\
1\end{array}$ & $\begin{array}{l}0.000 \\
1\end{array}$ & 0.78 \\
\hline Condición corporal & $2.50 \pm 0.03$ & $2.63 \pm 0.03$ & $2.27 \pm 0.04$ & 0.24 & 0.24 & 0.96 \\
\hline Calcio, $\mathrm{mmol} \mathrm{L}^{-1}$ & $2.08 \pm 0.16^{\mathrm{a}}$ & $1.73 \pm 0.16^{\mathrm{b}}$ & $1.80 \pm 0.21^{\mathrm{a}}$ & 0.001 & 0.001 & 0.86 \\
\hline $\begin{array}{l}\text { Ácidos grasos no } \\
\text { esterificados, mmol L- } \\
1\end{array}$ & $\begin{array}{l}0.410 \\
0.03^{\mathrm{a}}\end{array}$ & $\begin{array}{l}0.272 \\
0.03^{\mathrm{b}}\end{array}$ & $\begin{array}{l}0.394 \\
0.04^{\mathrm{a}}\end{array}$ & 0.01 & $\begin{array}{l}0.000 \\
1\end{array}$ & 0.92 \\
\hline Insulina, $\mathrm{ng} \mathrm{mL}^{-1}$ & $0.647 \pm 0.59$ & $0.654 \pm 0.59$ & $0.722 \pm 0.76$ & 0.34 & 0.26 & 0.76 \\
\hline
\end{tabular}

Cuadro 2. Producción de leche, condición corporal, y concentraciones de ácidos grasos no esterificados (AGNE), $\mathrm{Ca}^{2+}$ e insulina en suero sanguíneo en vacas HolsteinFriesian muestreadas $-30,-20,-10,-3,-2, y-1$ días preparto $(n=243)$, el día del parto, y $1,2,3,10,20$ y 30 días postparto $(n=250)$ en tres establos comerciales.

${ }_{\mathrm{a}, \mathrm{b}}$ Medias en la misma hilera con diferente superíndice difieren significativamente $(\mathrm{p}<0.05)$.

Los umbrales que se asociaron con pérdida de leche o leche no cosechada fueron con $\mathrm{Ca}^{2+} \leq 2.1$ mmol L ${ }^{-1}, \mathrm{AGNE} \geq 0.5 \mathrm{mmol} \mathrm{L}^{-1}$, e insulina con $\leq 0.26$, en el día 10 preparto. Los modelos de control de producción presentaron estimadores e interacciones entre $\mathrm{CC}$ y concentraciones preparto de metabolitos que fueron no significativos en ninguno de los casos ( $\mathrm{p}>0.36)$.

Cuando los niveles séricos de $\mathrm{Ca}^{2+}$ AGNE, e insulina (dicotomizados en el umbral seleccionado como óptimo) se combinaron en el mismo modelo en el día 10 previo al parto, solamente los AGNE $\geq 0.5 \mathrm{mmol} \mathrm{L}^{-1}$ (estimador $=-1.5 \pm 0.8 \mathrm{~kg} \mathrm{~d}^{-1} ; \mathrm{p}<0.01$ ) e insulina $\leq 0.30 \mathrm{ng}$ $\mathrm{mL}^{-1}$ (estimador $=-1.6 \pm 0.2 \mathrm{~kg} \mathrm{~d}^{-1} ; \mathrm{p}<0.0001$ ) permanecieron en el modelo después de controlar los efectos de fecha de parto, días en leche (lineal y cuadrático) y CC. Cuando se 
estratificó por fecha de parto, únicamente las concentraciones séricas de AGNE permanecieron en el modelo.

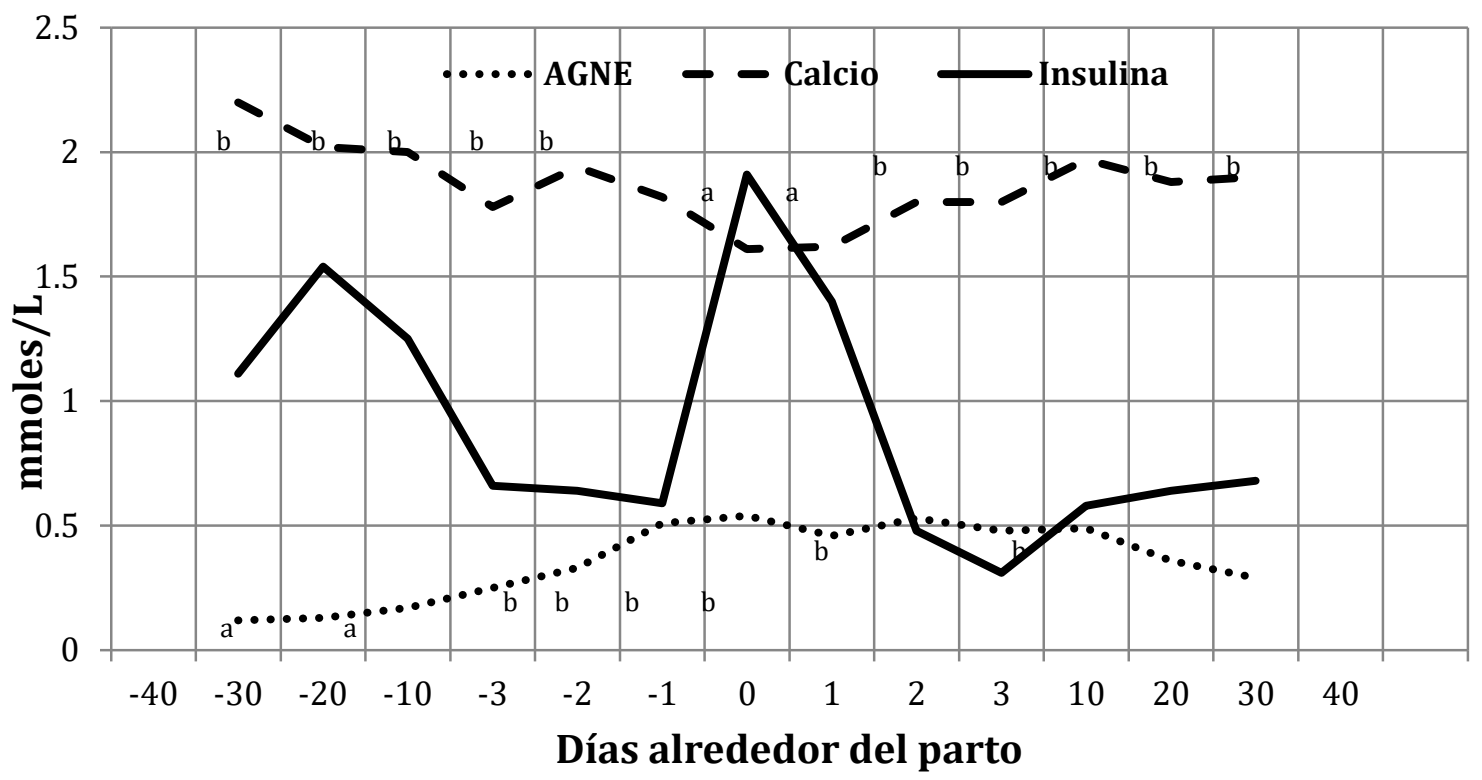

Figura 1. Concentraciones séricas de ácidos grasos no esterificados (AGNE), calcio e insulina antes, durante y después del parto de vacas Holstein-Friesian que consumen dietas completas de sorgo-soya en condiciones de confinamiento. Cada metabolito u hormona se probó con un modelo distinto. ${ }^{a b}$ Medias en la misma línea con diferente superíndice difieren significativamente $(p<0.5)$.

Cuando los niveles séricos de $\mathrm{Ca}^{2+}$, AGNE, e insulina se determinaron en el día 10 preparto y se relacionaron con los niveles obtenidos en el día 10 postparto (dicotomizados en el umbral seleccionado como óptimo), y se combinaron en el mismo modelo, solamente $\mathrm{Ca}^{2+}$ (estimador $=$ $1.2 \pm 0.6 ; p<0.001$ ), AGNE (estimador $=-2.0 \pm 0.8 \mathrm{~kg} \mathrm{~d}^{-1} ; \mathrm{p}<0.01$ ), e insulina (estimador = $\left.1.1 \pm 0.5 \mathrm{~kg} \mathrm{~d}^{-1} ; p<0.01\right)$ permanecieron en el modelo después de remover los efectos de días en leche, $\mathrm{CC}$ y fecha de parto. En los casos donde $\mathrm{Ca}^{2+}$, AGNE, e insulina se cuantificaron en los días 10 preparto, y 20 postparto (dicotomizados en el umbral seleccionado como óptimo), y se combinaron en el mismo modelo, únicamente $\mathrm{Ca}^{2+}$ (estimador $=-1.6 \pm 0.30 ; \mathrm{p}<0.001$ ), AGNE (estimador $=-1.7 \pm 0.4 \mathrm{~kg} \mathrm{~d}^{-1} ; \mathrm{p}<0.03$ ), e insulina (estimador $=-1.9 \pm 0.6 \mathrm{~kg} \mathrm{~d}^{-1} ; \mathrm{p}<0.01$ ) medidos 10 día postparto permanecieron en el modelo después de controlar los efectos de días en leche, CC y fecha de parto.

El Cuadro 3 muestra la proporción de animales en el umbral de metabolitos de alto riesgo, los coeficientes de disparidad y los intervalos de confianza por umbrales. En el día 10 preparto, los 
coeficientes de disparidad (CD) más altos fueron cuando $72.11 \%$ estuvieron por debajo del umbral de $\mathrm{Ca}^{2+}[(\mathrm{CD}=2.2$; intervalo de confianza (IC) del $95 \%=1.8-2.28)], 67.40 \%(\mathrm{CD}=0.8 ; \mathrm{IC}=$ 0.30 - 1.14) estuvieron por debajo del umbral AGNE; mientras que insulina $6.02 \%$ de vacas estuvieron en el grupo de riesgo alto de insulina $(\mathrm{CD}=0.8 ; \mathrm{IC}=1.61-1.90)$.

En el día 10 postparto, los CD más altos fueron cuando $66.41 \%$ de las vacas estuvieron abajo del umbral de $\mathrm{Ca}^{2+}(\mathrm{CD}=1.4$; IC $=0.90-2.60) ; 22.0 \%$ con AGNE $(\mathrm{CD}=0.9$; $\mathrm{IC}=0.89-$ 2.13); mientras que $29.7 \%$ de animales estuvieron por debajo del nivel de riesgo de insulina (CD $=0.7 ; \mathrm{IC}=0.7-2.01)$. En el d 20 postparto, los CD más altos fueron cuando $40.0 \%$ de los animales estuvieron por debajo del umbral de $\mathrm{Ca}^{2+}(\mathrm{CD}=2.5$; IC $=1.8-2.90), 20.0 \%$ de las vacas estuvieron por debajo de $\mathrm{AGNE}(\mathrm{CD}=0.5$; $\mathrm{IC}=0.50-1.31)$; mientras que $15.12 \%$ de animales estuvieron en el grupo de riesgo alto $(\mathrm{CD}=0.9 ; \mathrm{IC}=1.81-2.2)$ para insulina.

\begin{tabular}{|l|l|l|l|l|}
\hline Ítem & $\begin{array}{l}\text { Proporción de } \\
\text { animales en el } \\
\text { umbral de } \\
\text { metabolitos de } \\
\text { riesgo alto (\%) }\end{array}$ & $\begin{array}{l}\text { Coeficiente } \\
\text { de } \\
\text { disparidad }\end{array}$ & $\begin{array}{l}\text { Intervalos de } \\
\text { confianza de } \\
\text { Wald al 95\% }\end{array}$ & $P$ \\
\hline \multicolumn{5}{|l|}{ Preparto vs día 10 posparto } \\
\hline Calcio $\leq 2.1 \mathrm{mmol} \mathrm{L}^{-1}$ & 72.11 & 2.2 & $1.8-2.28$ & 0.05 \\
\hline AGNE $\geq 0.5 \mathrm{mmol} \mathrm{L}^{-1}$ & 67.40 & 0.8 & $0.30-1.4$ & $<0.06$ \\
\hline Insulina $\leq 0.26 \mathrm{ng} \mathrm{mL}^{-1}$ & 6.02 & 0.8 & $0.61-0.90$ & $<0.005$ \\
\hline Preparto vs día 20 posparto & \multicolumn{5}{l|}{} \\
\hline Calcio $\leq 2.1 \mathrm{mmol} \mathrm{L}^{-1}$ & 66.41 & 1.4 & $0.90-2.60$ & 0.01 \\
\hline AGNE $\geq 0.5 \mathrm{mmol} \mathrm{L}^{-1}$ & 22.00 & 0.9 & $0.89-2.13$ & 0.05 \\
\hline Insulina $\leq 0.26 \mathrm{ng} \mathrm{mL}^{-1}$ & 29.70 & 0.7 & $0.7-2.1$ & 0.05 \\
\hline
\end{tabular}

Cuadro 3. Asociación entre las concentraciones sanguíneas de Calcio, AGNE e insulina muestreadas a los últimos 10 días $(n=243)$ preparto y su relación con producción de leche $\left(\mathrm{kg} \mathrm{d}^{-1}\right)$ a los 10 y 20 días $(\mathrm{n}=251)$ postparto de vacas HolsteinFriesian en tres establos comerciales.

${ }^{1}$ Cada metabolito u hormona se probó en un modelo separado. Las concentraciones de metabolitos u hormonas fueron dicotomizados a diferentes umbrales y únicamente los umbrales con la más alta asociación con pérdida de leche fue presentada. Controlado por el efecto linear y cuadrático de días en leche, fecha de parto y el efecto aleatorio de establo. ${ }^{3}$ Controlado por el efecto linear y cuadrático de días en leche, fecha de parto y el efecto aleatorio de establo. 


\section{Discusión}

El objetivo del presente estudio fue relacionar las concentraciones séricas de $\mathrm{Ca}^{2+}$, AGNE e insulina 10 días antes del parto con la PL a los 10 y 20 días de lactancia, para cuantificar el riesgo de perder leche en vacas Holstein-Friesian en condiciones de confinamiento. El propósito central es encontrar un valor o un rango de valores de metabolitos que permitan predecir las posibles pérdidas de leche en el inicio de la lactancia. Con esta idea en mente, se realizaron muestreos de sangre antes, durante y después del parto en vacas provenientes de tres establos (Cuadro 2).

La PL en estudio se obtuvo de una producción diaria promedio de aproximadamente 26.00 a $30.00 \mathrm{~kg}$ entre los días 10 y 20 de lactancia, alcanzando a los 100 días un promedio de $37.33 \pm$ 5.60. Esto concuerda con el estudio realizado por Ponce (2011), quién señaló que vacas de alta producción en sistemas intensivos de la Laguna logran una PL promedio de $31.73 \pm 3.40$, que es similar a lo observado en el presente estudio. Los rendimientos lecheros de 23.22 y $22.90 \%$ mayores del establo tres, con conexión a uno y dos, respectivamente, quizás se expliquen por las diferencias en la calidad del alimento. Las dietas de los establos uno y dos tuvieron concentraciones más bajas de proteína y FDN. Dicho efecto también puede deberse a la frecuencia de alimentación y al manejo general de la alimentación.

Dixon (1999) y Olsen et al. (1999) reportaron que la alimentación con concentraciones mayores de proteína pueden incrementar la digestión in situ de la fibra y la materia orgánica. Olsen et al. (1999) utilizaron novillos con dietas basada en heno de forrajes (4.9\% PC) para determinar si la infusión ruminal de proteína cruda afectaba la degradación de la fibra in situ. La diferencia en proteína fue alcanzada con la infusión de caseinato de sodio en el rumen a la tasa de 0.0, 0.03, 0.06, 0.09, ó $0.12 \%$ del PV inicial. Asumiendo que no hubo diferencias en el CMS o PV, esto debería igualar el incremento en el consumo de proteína de 0.0, 1.70, 3.40, 5.10, ó 6.80 de unidades porcentuales. Conforme el nivel de proteína incrementa, la degradación in situ de la materia seca y orgánica de los forrajes también incrementa. También se observaron incrementos en la producción de amoniaco y ácidos grasos volátiles (AGV) en el rumen. Incrementando la proteína cruda de la dieta de 11.60 a15.00 \% se incrementa la digestibilidad de la proteína cruda en el tracto total de 54.3 a $60.6 \%$ en la semana tres a dos preparto en vacas lecheras (Doreau et al. 1990).

La diferencia en PL a favor del establo tres también se puede deber a la frecuencia de alimentación, debido a que en éste establo el alimento se ofreció tres veces por día (33.30 \% cada vez), mientras que los establos uno y dos se ofrecieron solamente en dos porciones (50.00\% cada

No 19, Vol. 9 (2), 2017. ISSN 2007 - 0705, pp.: 306 - 328 
vez), sugiriendo la posibilidad de un uso más eficiente del alimento con el mayor número de ofrecimientos (Cuadro 1). Hart el al. (2014) observaron que las vacas que recibieron el alimento del día en tres porciones ( $33.33 \%$ cada vez) consumieron más materia seca que las alimentadas una (100.00\%) o dos veces al día (50.00\% cada vez). La frecuencia de alimentación no tuvo efectos en PL, composición o eficiencia de PL. Por lo tanto, los nutrientes consumidos cuando se ofrece tres veces por día pudieron haberse metabolizados para reservas corporales y no tanto para síntesis de leche. La frecuencia de alimentación tuvo poco efecto en el comportamiento alimenticio, sin embargo, alteró la magnitud del consumo de materia seca.

En el presente estudio, la CC preparto y posparto fue similar en los establos y no se relacionó con la PL. Esto se atribuyó a que la CC se estratificó con rangos de valores estrechos y por debajo de 2.50. La proporción de vacas gordas fue $2.1 \%$ y se consideró como inexplicable para las condiciones de producción del área del estudio. Dicha proporción, posiblemente, pudo haber sido mayor si las vacas se hubieran monitoreado en los últimos días previos al parto. Los resultados obtenidos en el presente estudio son similares a los observados por otros; donde las relaciones entre CC y PL fueron no significativas (Ruegg y Milton, 1995; Berry et al. 2007); o débiles (Locker et al. 2012; Green et al. 2014). Por el contrario, otros estudios demostraron relaciones mayores y positivas entre CC y PL (Roche et al. 2007; Sheehy et al. 2017) cuando se consideraron vacas lecheras en la transición del parto a la lactancia.

Las concentraciones séricas de $\mathrm{Ca}^{2+}$ encontradas en el presente estudio fueron de aproximadamente $1.87 \mathrm{mmol} \mathrm{L}^{-1}$, con un rango de variación amplia desde 2.20 hasta $1.61 \mathrm{mmol} \mathrm{L}^{-}$ ${ }^{1}$ en el día de parto. Posteriormente, incrementaron a $1.90 \mathrm{mmol} \mathrm{L}^{-1} 30$ días posparto (Figura 1). Como se indicó, anteriormente, el umbral óptimo de $\mathrm{Ca}^{2+}$ se estableció en $<2.1 \mathrm{mmol} \mathrm{L}^{-1}$. $\mathrm{El} \mathrm{Ca}^{2+}$ absorbido requerido por $\mathrm{kg}$ de leche producido es de $0.22 \mathrm{~g}$ para vacas Holstein-Friesian (NRC, 2001). En el presente estudio, la cantidad de leche perdida por $\mathrm{Ca}^{2+}$ por debajo del umbral fue de 2.2 a $1.4 \mathrm{~kg}$ de leche por animal ${ }^{-1}$ día $^{-1}$; cuando $\mathrm{Ca}^{2+}$ se cuantificó 10 días antes del parto y se relacionó con PL a los 10 y 20 días posparto, respectivamente (Cuadro 2). Dicha disminución quizás se deba a la movilidad de las reservas corporales y a la participación del mineral en el proceso de formación de leche. De acuerdo a los requerimientos establecidos por el NRC se observó una deficiencia de $\mathrm{Ca}^{2+}$, lo que obliga al animal a extraer $\mathrm{Ca}^{2+}$ de los huesos para mantener la homeostasis del fluido extracelular. La deficiencia en los requerimientos del $\mathrm{Ca}^{2+}$ predispone a los animales a problemas de osteoporosis y osteomalacia en los huesos, lo que aumenta el riesgo a 
fracturas, sin embargo, esto no fue observado en el presente estudio.

Chapinal et al. (2012b) indicaron que el $73.00 \%$ de las vacas de establos de USA y Canadá perdieron $3.80 \mathrm{~kg}_{\text {animal }}{ }^{-1} \mathrm{~d}^{-1}$ de leche cuando se consideró el hato completo, y alrededor de 2.90 $\mathrm{kg}$ cuando se consideraron únicamente las vacas adultas. En otros estudios, Chapinal et al. (2012a) reportaron reducciones de leche similares a las observadas en el presente estudio; los mismos autores concluyeron que las concentraciones de $\mathrm{Ca}^{2+}$ bajas en la semana previa al parto, se asociaron con leche perdida o leche no cosechada en los primeros cuatro días postparto; después de éste tiempo la relación entre las concentraciones de $\mathrm{Ca}^{2+}$ y PL tienden a ser mínimas; posiblemente, por una restauración del balance homeostático del mineral debido al restablecimiento del consumo de alimento y el BEN, como se observó en el presente estudio, alrededor del día 30 posparto.

Uno de los problemas recurrentes en los establos lecheros modernos es la presencia de hipocalcemia. Sin embargo, es todavía más impactante la hipocalcemia subclínica que se presenta en concentraciones de 1.38 a $2.00 \mathrm{mmol} \mathrm{L}^{-1}$ (Goff, 2008). Esto permite suponer que, en el presente estudio, una proporción de las vacas (aproximadamente el 66.0\%) tuvieron algún padecimiento relacionado con la deficiencia de $\mathrm{Ca}^{2+}$. Si dicha indicación es correcta, existe la oportunidad de usar los valores de $\mathrm{Ca}^{2+}$ para prevenir no sólo la perdida de leche sino también la presencia de hipocalcemias subclínicas.

Con respecto a AGNE, en el presente estudio, el valor promedio fue de $0.35 \mathrm{mmol} \mathrm{L}^{-1}$ con un rango entre 0.18 y $2.24 \mathrm{mmol} \mathrm{L}^{-1}$. Dicho valor promedio corresponde al límite inferior descrito por Meléndez et al. (2002), quienes establecen que la concentración máxima de AGNE al parto es $1.2 \mathrm{mmol} \mathrm{L}{ }^{-1}$ en un rango de $0.9-1.2 \mathrm{mmol} \mathrm{L}^{-1}$. En el presente estudio, la leche perdida por el incremento en los niveles de AGNE fue 0.8 y $0.7 \mathrm{~kg}_{\text {de }}$ leche animal ${ }^{-1}$ día $^{-1}$; para los días 10 y 20 de lactancia, respectivamente. El incremento en los niveles de AGNE se puede explicar por el incremento en los requerimientos nutricionales de las vacas o que éstas estuvieron en BEN y que movilizaron lípidos para cubrir sus necesidades de energía (Bernabucci et al. 2005). Sin embargo, como se indicó anteriormente, el incremento en los AGNE va acompañado con el incremento de varios metabolitos y hormonas, tal vez uno de los más relevantes es $\beta \mathrm{HB}$. Sin embargo, el incremento en AGNE y $3 \mathrm{HB}$ va acompañado por una reducción en la glucosa sanguínea. En condiciones de concentraciones elevadas de $\beta \mathrm{HB}$, seguido de la reducción de glucosa, posiblemente, haya una reducción en la gluconeogénesis y en el sistema inmune de las vacas, con 
la consecuente reducción en la PL. Zarrin et al. (2017) observaron que la glucosa sanguínea se reduce debido a la infusión de $\beta \mathrm{HB}$ en el pre- y posparto de vacas lecheras en transición. En ambos periodos, dicho efecto no fue idénticamente mediado por insulina. El incremento en la hipoglucemia por cantidades exógenas de BHB después del parto supone una habilidad regulatoria intrínseca en el metabolismo de la glucosa. Un posible incremento de la desaparición de BHB después del parto asume una adaptación a numerosos tejidos involucrados en el metabolismo de lípidos y carbohidratos (Zarrin et al. 2013).

En asociación con estos eventos, al mismo tiempo, se presenta una demanda alta por glucosa para la formación de lactosa y síntesis de leche en el inicio de la lactancia, que comúnmente se piensa que es la principal causa de las concentraciones bajas de glucosa, en adición a la tasa insuficiente de gluconeogénesis para llenar las demandas de glucosa después del parto.

En el presente estudio, las concentraciones séricas de AGNE incrementaron conforme los animales se acercaban al parto y se redujeron aproximadamente 20 días después del parto (Figura 1). VandeHaar et al. (1999) también observaron que la concentración sérica de AGNE incremento conforme se acercaba al tiempo de parto; éste incremento es, comúnmente, observado y es probablemente relacionado con la disminución en el CMS y los cambios hormonales asociados con el parto y la lactogénesis. Bertics et al. (1992) redujeron la magnitud de los AGNE incrementando la alimentación forzada de las vacas durante el periodo de transición preparto, sin embargo, no fue posible eliminarlo completamente. Los resultados obtenidos en el presente estudio son similares a los observados por Chapinal et al. (2011) y Chapinal et al. (2012a, 2012b). Otros reportes (Ospina et al. 2010a, 2010b) indican que AGNE preparto mayores $\left(\geq 0.33 \mathrm{mEq} \mathrm{L}^{-1}\right)$ resultaron en una reducción de aproximadamente $683.00 \mathrm{~kg}$ de leche animal ${ }^{-1}$ lactancia $^{-1}$.

Sin embargo, con un nivel todavía más alto $\left(\geq 0.57 \mathrm{mEq} \mathrm{L}^{-1}\right)$ la PL en lugar de disminuir, observó un incrementó de aproximadamente $488.00 \mathrm{~kg}_{\text {animal }}{ }^{-1}$ por lactancia.

Los datos obtenidos en el presente estudio y los reportados en la literatura (Bicalho et al., 2017) magnifican la importancia de AGNE en la PL en el inicio de la lactancia. Sin embargo, todavía se está lejos de obtener un valor previo al parto que permita definir si habrá una reducción en la PL. Sin embargo, de acuerdo con el presente estudio, es posible afirmar que $67.40 \%$ las vacas estuvo por debajo del umbral óptimo de $0.5 \mathrm{mmol} \mathrm{L}^{-1}$ y que dichos valores se relacionaron con pérdidas de aproximadamente $0.8 \mathrm{~kg} \mathrm{anmal}^{-1} \mathrm{día}^{-1}$. Los resultados obtenidos en el presente estudio son similares a otros reportados en la literatura. Marín et al. (2011) concluyeron que las 
concentraciones de AGNE se relacionaron cuadráticamente con PL a los 100 días de lactancia. Las vacas con niveles tanto bajos y altos de AGNE al parto, tuvieron una producción más baja que los niveles intermedios.

Las relaciones entre la deficiencia de $\mathrm{Ca}^{2+}$ y el incremento de AGNE al parto fueron demostradas por Chamberlain et al. (2013), quienes observaron que la deficiencia de $\mathrm{Ca}^{2+}$ se asoció con un incremento en los niveles de AGNE al parto, y un incremento en la deposición de lípidos en el hígado a los siete y 35 días en leche en un grupo de vacas positivas a hipocalcemia. La concentración de $\mathrm{Ca}^{2+}$ al parto se asoció con los AGNE en los días -14, 7 y 21 alrededor del parto; lo que enfatiza la relación de dependencia entre los AGNE y $\mathrm{Ca}^{2+}$ en vacas lecheras durante el periodo de transición.

En el presente estudio, la concentración promedio de insulina fue de $0.674 \mathrm{ng} \mathrm{mL}^{-1}$, y varió de 0.29 a $2.26 \mathrm{ng} \mathrm{mL}^{-1}$, demostrando una variabilidad alta entre concentraciones. Los valores de insulina por debajo de $0.26 \mathrm{ng} \mathrm{mL}^{-1}$ mostraron perdidas de leche de aproximadamente 0.80 y 0.70 $\mathrm{kg}_{\text {animal }}{ }^{-1} \mathrm{~d}^{-1}$; para los días 10 y 20 de lactancia, respectivamente. Dichas perdidas quizás se deban que la insulina es una hormona anti-lipolítica potente y que la reducción en su concentración sanguínea incrementaría la lipolisis en el tejido adiposo, promoviendo incrementos de AGNE (Dimitriadis et al., 1997).

Como se mencionó anteriormente, los incrementos de AGNE después del parto se explican, principalmente, por el contenido de grasa hepática contenida en las vacas. Sin embargo, con el inicio de lactancia, se requieren grandes cantidades de glucosa para PL. Por lo tanto, en adición al incremento de la glucosa endógena, la misma glucosa es distribuida reduciendo su oxidación y tanto AGNE como ßHB son usados como sustratos productores de energía (Drackley et al., 2001). Las concentraciones elevadas de AGNE y BHB son usualmente acompañadas de una reducción de glucosa e insulina posparto, lo que se refleja en la demanda de glucosa por el inició de la lactancia y BEN.

La insulina ha demostrado que suprime la producción de glucosa a través de la inhibición de enzimas reguladoras involucradas en la gluconeogénesis (Hayirli, 2006). Sin embargo, en dicha acción también participa el ßHB (Zarrin et al. 2013). La baja actividad de las enzimas gluconeogénicas y la baja tasa gluconeogénica posparto son las razones principales de la baja concentración de glucosa en plasma (Aschenbach et al. 2010).

Aparentemente, los niveles de insulina tienden a reducirse durante las primeras semanas de 
Relaciones entre calcio, ácidos grasos no esterificados, e insulina sanguínea en preparto y leche bovina perdida en el inicio de la lactancia

lactancia, lo que posiblemente, se deba a la cantidad de glucosa en sangre y al consumo de materia seca (Bicalho et al., 2017). En apoyo a lo anterior, en un estudio de dosis y respuestas, Spicer et al. (2002) observaron una correlación positiva entre insulina y glucosa $(\mathrm{r}=0.35)$ y entre consumo de materia seca e insulina $(r=0.37)$. También se observó una correlación positiva entre los consumos de materia seca y glucosa $(\mathrm{r}=0.44)$ en las primeras 12 semanas de lactancia en vacas Holstein-Friesian, lo que sugiere una dependencia de la insulina con consumo de alimento.

Mann et al. (2016) indicaron que las vacas que recibieron dietas concentradas en energía durante el periodo seco mostraron un grado de lipogénesis preparto mayor y no observaron diferencias en la actividad de las enzimas lipolíticas comparadas con las vacas alimentadas de acuerdo a los estándares. Las concentraciones básicas de insulina decrecieron, mientras que los AGNE incrementaron en el posparto en el grupo sobrealimentado con energía. Las diferencias en AGNE entre vacas sobrealimentadas en el preparto y las concentraciones séricas de ßHB altas no deberían atribuirse a cambios en las señales de insulina en el tejido adiposo; sin embargo, pueden asociarse con concentraciones bajas de insulina y glucosa, reflejando un déficit más severo de energía en estas vacas. Desafortunadamente, en el presente estudio no se determinó ßHB y no hubo oportunidad de relacionar los resultados del presente estudio con cetosis.

Los estudios de dependencia de insulina con respecto a glucosa, AGNE u otros metabolitos se hacen con el uso de braquetes euglucémicos-hiperinsulinémicos. Con este respecto, Butler et al. (2004) demostraron que la infusión de insulina disminuyó las concentraciones de AGNE en 68.00\% comparado con los niveles básicos. Al mismo tiempo, otro grupo de vacas que no recibieron insulina, mantuvieron las concentraciones séricas de AGNE en $96.00 \%$ en comparación con los niveles básicos. Los autores concluyeron que la PL no se alteró; sin embargo, la grasa y proteína de la leche se redujeron 27\%. Considerando que insulina tiene un papel determinante en el metabolismo de carbohidratos y que sus concentraciones pueden determinar el nivel energético del animal, es posible sugerir su uso en la predicción de PL en la lactancia siguiente.

Los resultados del presente estudio sugieren que los valores de $\mathrm{Ca}^{2+}$ bajos y AGNE altos determinados diez días antes del parto, son herramientas útiles para predecir perdidas en PL al inicio de la lactancia. En términos de prioridad, los coeficientes de disparidad indican una mayor efectividad de la concentración de $\mathrm{Ca}^{2+}$ con respecto a los niveles altos de AGNE (Cuadro 3). Sin embargo, dichos niveles de AGNE también fueron efectivos para prevenir perdidas al inicio de la lactancia, y después los valores de insulina. La razón del uso de uno u otro dependerá del costo y

No 19, Vol. 9 (2), 2017. ISSN 2007 - 0705, pp.: 306 - 328 
la facilidad de análisis. Dependiendo de la situación geográfica del hato lechero la determinación más común es la concentración de $\mathrm{Ca}^{2+}$ sanguíneo.

Otros factores como la composición y calidad de las dietas ofrecidas determinaron las concentraciones séricas de metabolitos y hormonas mediante la formación de sustratos promovido por el estado fisiológico de los animales. Como se puede ver en los resultados, las diferencias entre establos fueron debidas a la frecuencia de alimentación y seguramente a otros factores no controlados en la presente investigación.

También se observó que la leche obtenida es la resultante de varios factores incluyendo la vaca, el manejo y el ambiente. La variabilidad de dichos factores es grande no sólo entre animales sino también entre componentes que influyen en la obtención de producto. Por lo que la precisión del valor predicho para los metabolitos u hormona estudiados estará expuesta a las condiciones propias de los hatos estudiados. Sin embargo, la determinación de alguno de los metabolitos o de insulina, le ayudarán al productor a predecir la leche que perderá o dejará de cosechar al inicio de la lactancia. Lo que puede prevenir con un muestreo rápido de las vacas que a la vista pudieran resultar susceptibles de las pruebas. También pudiera ser que de un establo sólo se tomará una muestra pequeña (aproximadamente 30 vacas) y con dicho nivel de $\mathrm{Ca}^{2+}$, AGNE o insulina, predecir la ganancia en PL.

\section{Conclusiones}

Los datos indican que una alta proporción de las vacas están deficientes en calcio, ácidos grasos no esterificados e insulina cuando sus valores se determinan 10 días antes de parto, y que la leche perdida o no cosechada está asociada con el metabolismo del calcio y ácidos grasos no esterificados que son regulados por insulina. La deficiencia de calcio produce pérdidas mayores de leche que los excesos de ácidos grasos no esterificados y las concentraciones séricas de insulina, cuando se determinan 10 días antes de parto y la leche se estima en los primeros 10 y 20 días de lactancia. El calcio sérico es el mejor predictor de pérdidas de leche en comparación con ácidos grasos no esterificados e insulina en las vacas en el periodo previo a la lactancia.

\section{Agradecimientos}

Los autores agradecen ampliamente a Biotecap, S.A. de C. V., en especial al Ing. Juan de Dios Roldán y el M.C. Adelfo Vite por proveer las facilidades para realizar la fase de campo del presente 
Relaciones entre calcio, ácidos grasos no esterificados, e insulina sanguínea en preparto y leche bovina perdida en el inicio de la lactancia

estudio. El agradecimiento es extensivo a los propietarios y encargados de los establos donde se llevó a cabo este proyecto.

\section{Referencias}

AOAC. (2006). Association of Official Analytical Chemists. Official methods of analysis. 18th. (Ed.). Association of Official Analytical Chemists Press. Gaithersburg, MD. AOAC International.

Aschenbach, J.R., Kristensen, N. B., Donkin, S.S., H.M. Hammon, H.M., Penner, G. B. (2010). Gluconeogenesis in dairy cows: The secret of making sweet milk from sour dough. IUBMB Life 62:869-877.

Berry, D.P., Lee, J. M., McDonald, K.A., Roche, J.R. (2007). Body condition score and body weight effects on dystocia and stillbirths and consequent effects on postcalving performance. Journal of Dairy Science 90: 4201- 4211.

Bernabucci, U., Ronchi, B., Lacetera, N., Nardone, A. (2005). Influence of body condition score on relationships between metabolic status and oxidative stress in periparturient dairy cows. Journal of Dairy Science 88: 2017-2026.

Bertics, S.J., Grummer, R.R., Cadorniga-Valino, C., Stoddard, E.E. (1992). Effect of prepartum dry matter intake on liver triglyceride concentration and early lactation. Journal of Dairy Science. 75:1914-1922.

Bicalho, M. L.S., Santin, T. Rodrigues, M.X., Marques C.E., Lima S.F., Bicalho, R.C. (2017). Dynamics of the microbiota found in the vaginas of dairy cows during the transition period: Associations with uterine diseases and reproductive outcome. Journal of Dairy Science. 100: 3043-3058.

Butler, S.T., Pelton, S.H., Butler, W.R. (2004). Insulin increases17ß-estradiol production by the dominant follicle of the first postpartum follicle wave in dairy cows. Reproduction. 127: 537- 45.

Chamberlain, W.G., Middleton, J.R., Spain, J.N., Johnson, G.C., Ellersieck, M.R., Pithua, P. (2013). Subclinical hypocalcaemia, plasma biochemical parameters in postparturient dairy cows. Journal of Dairy Science. 96: 7001-7013.

Chapinal, N., Carson, M.E., LeBlanc, S.J., Leslie, K.E., Godden, S., Capel, M., Santos, J.E.P., Overton, M.W., Duffield, T.F. (2012a). The association of serum metabolites in the 
transition period with milk production, and early-lactation reproductive performance. Journal of Dairy Science. 95: 1301-1309.

Chapinal, N., Carson, M.E., Duffield, T.F., Capel, M., Godden, S., Overton, M.W., Santos, J.E.P., LeBlanc, S.J. (2011). The association of serum metabolites with clinical disease during the transition period. Journal of Dairy Science. 94: 489- 4903.

Chapinal, N., LeBlanc, S.J., Carson, M.E., Leslie, K.E., Godden, S., Capel, M., Santos, J.E.P., Overton, M.W., Duffield, T.F. (2012b). Herd-level association of serum metabolites in the transition period with disease, milk production, and early lactation reproductive performance. Journal of Dairy Science. 95: 5676-5682.

Dimitriadis, G., Parry-Billings, M., Challiss, R.A.J., Wegener, G., Newsholme, E.A. (1997). The effects of insulin on transport and metabolism of glucose in skeletal muscle from hypothyroid rats. European Journal of Clinical Investigation. 27: 475- 483.

Dixon, R.M. (1999). Effects of addition of urea to a low nitrogen diet on rumen digestion of a range of roughages. Australian Journal Agricultural Research. 50:1091-1097.

Doreau, M., Delacroix, A., Jouany, J.P., Durier, C., Remond, B. (1990). The influence of physiological state and dietary nitrogen supply on digestion in the dairy cow. Journal of Animal Science. 68:3853 - 3860.

Drackley, J.K., Overton, T.R., Douglas, G.N. (2001). Adaptations of glucose and long-chain fatty acid metabolism in liver of dairy cows during the periparturient period. Journal of Dairy Science. 84:E100-E112.

Duffield, T.F., Lissemore, K.D., McBride, B.W., Leslie, K.E. (2009). Impact of hyperketonemia in early lactation dairy cows on health and production. Journal of Dairy Science. 92:571580.

García, E. (2005). Modificaciones al sistema de clasificación climática de Köppen. Instituto de Geografía. UNAM. México. $\quad$ ta. $\quad$ ed. $\quad$ p. 20.http://www.igeograf.unam.mx/sigg/utilidades/docs/pdfs/publicaciones/geo_siglo21/ser ie_lib/modific_al_sis.pdf. Consultada el 25 de noviembre de 2014.

Goering, H.K., Van Soest, P.J. (1970). Forage fibber analyses (Apparatus, reagents, procedures, and some applications). Agriculture Handbook No. 379. ARS-USDA, Washington DC, USA. ARS-USDA Press Inc. 
Relaciones entre calcio, ácidos grasos no esterificados, e insulina sanguínea en preparto y leche bovina perdida en el inicio de la lactancia

Goff, J.P. (2008). Transition cow immune function and interaction with metabolic diseases. Pages 45-57 in Proc. $17^{\text {th }}$ Annual Tri-State Dairy Nutrition Conference. The Ohio State University, Columbus.

Green, L.E., Huxley, J.N., Banks, M.J., Green, C. (2014). Temporal associations between low body condition, lameness and milk yield in a UK dairy. Preventive Veterinary Medicine. 113: 63 $-71$.

Gross, J., van Dorland, H.A., Bruckmaier, R.M., Schwarz, F.J. (2011). Performance and metabolic profile of dairy cows during a lactational and deliberately induced negative energy balance with subsequent realimentation. Journal of Dairy Science. 94:1820-1830.

Hart, K.D., McBride, B.W., Duffield, T.F. DeVries, T. J. (2014). Effect of frequency of feed delivery on the behavior and productivity of lactating dairy cows. Journal of Dairy Science. 97:1713 - 1724.

Hayirli, A. (2006). The role of exogenous insulin in the complex of hepatic lipidosis and ketosis associated with insulin resistance phenomenon in postpartum dairy cattle. Veterinary Research Communication. 30:749 - 774.

Horst, R.L., Goff, J.P., Reinhardt, T.A. (1994). Calcium and vitamin D metabolism in the dairy cow. Journal Dairy Science. 77:1936 -1951.

LeBlanc, S.J. (2010). Monitoring metabolic health of dairy cattle in the transition period. Journal of Reproduction and Development. 56(Suppl.): PS29 - PS35.

LeBlanc, S.J., Duffield, T.F., Leslie, K.E., Bateman, K.G., TenHag, J., Walton, J.S., Johnson, W. H. (2002). The effect of prepartum injection of vitamin $E$ on health in transition dairy cows. Journal of Dairy Science. 85: 1416 -1426.

Locker, S., Bastin, C., Miglior, F., Sewalem, A., Schaeffer, L.R., Jamrozik, J., Ali, A., Osborne, V. (2012). Genetic and environmental relationships between body condition score and milk production traits in Canadian Holsteins. Journal of Dairy Science. 95: 410 - 419.

Mann, S., Nydam, D.V., Abuelo, A., Leal-Yepes, F.A., Overton, T.R., Wakshlag, J.J. (2016). Insulin signaling, inflammation, and lipolysis in subcutaneous adipose tissue of transition dairy cows either overfed energy during the prepartum period or fed a controlled-energy diet. Journal of Dairy Science. 99:6737- 6752. 
Marín, M.P., Ríos, C., Contreras, H., Robles J., Meléndez, P. (2011). Ácidos grasos no esterificados al parto y su relación con producción lechera en vacas Holstein. Archivos de Zootecnia. 60: $257-264$.

Meléndez, P., Donovan, A., Risco, C. A., Hall, B. A., Littell, y R., Goff, J. (2002). Metabolic responses of transition cows fed anionic salts and supplemented at calving with calcium and energy. Journal of Dairy Science. 85: 1085-1092.

NRC, (2001). Nutrient Requirements of Dairy Cattle. 7th rev. ed. Natl. Acad. Sci., Washington, DC.

Olsen, K.C., Chochran R.C., Jones, T.J., Vanzant, E.S, Titgemeyer, E.C., Johnson, D.E. (1999). Effects of ruminal administration of supplemental degradable intake protein and starch on utilization of low-quality warm-season grass hay by beef steers. Journal of Animal Science. 77:1016-1025.

Ospina, P.A., Nydam, D.V., Stokol, T., Overton, T.R. (2010a). Evaluation of nonesterified fatty acids and $\beta$-hydroxybutyrate in transition dairy cattle in the northeastern United States: Critical thresholds for prediction of clinical diseases. Journal of Dairy Science 93: 546 554.

Ospina, P.A., Nydam, D.V., Stokol, T., Overton, T. R. (2010b). Associations of elevated nonesterified fatty acids and $\beta$-hydroxybutyrate concentrations with early lactation reproductive performance and milk production in transition dairy cattle in the northeastern United States. Journal of Dairy Science. 93: 1596 -1603.

Ponce, C. Ismael. (2011). Efecto de Sacharomyces cerevisiae en la producción y calidad de la leche de vacas Holstein-Friesian en condiciones de estrés calórico. Tesis de Maestría en Ciencias en Innovación Ganadera. Posgrado en Producción Animal. Universidad Autónoma Chapingo.

Reinhardt, T.A., Lippolis, J.D., McCluskey, B.J., Goff, J. P., Horst, R.L. (2011). Prevalence of subclinical hypocalcemia in dairy herds. Veterinary Journal. 188: 122-124.

Roche, J.R., Lee, J.M., McDonald, K.A., Berry, D.P. (2007). Relationships among body condition score, body weight, and milk production variables in pasture-based dairy cows. Journal of Dairy Science. 90: 3802- 3815.

Ruderman, N.B., Goodman, M. N. 1973. Regulation of ketone body metabolism in skeletal muscle. American Journal of Physiology. 224:1391-1397. 
Ruegg, P.L., Milton, R.L. (1995). Body condition scores of Holstein cows on Prince Edward Island, Canada: relationships with yield, reproductive performance, and disease. Journal of Dairy Science. 78: 552-564.

Schlumbohm, C., Harmeyer, J. (2003). Hypocalcemia reduces endogenous glucose production in hyperketonemic sheep. Journal of Dairy Science. 86:1953-1962.

Seifi, H.A., LeBlanc, S.J., Leslie, K.E., Duffield, T.F. (2011). Metabolic predictors of post-partum disease and culling risk in dairy cattle. Veterinary Journal. 188: 216 - 220.

Sheehy, M.R., Fahey, A.G., Aungier, S.P.M., Carter, F., Crowe, M.A., Mulligan, F.J. (2017). A comparison of serum metabolic and production profiles of dairy cows that maintained or lost body condition 15 days before calving. Journal of Dairy Science 100: 536 -547.

Spicer, L.J., Francisco, C.C., Jones, D., Waldner, D.N. (2002). Changes in plasma insulin and glucose during early lactation in Holstein cows. Oklahoma Agricultural Experiment Station. Animal Science Research Report. Department of Animal Science. Stillwater, OK. Statistical Analysis System (SAS). (2014). SAS/STAT User's Guide, Software Version 9.2. SAS Institute, Cary, N. C. USA.

VandeHaar, M.J., Yousif, G., Sharma, B.K., Herdt, T.H., Emery, R.S., Allen, M.S., Liesman, J. S. (1999). Effect of energy and protein density of prepartum diets on fat and protein metabolism of dairy cattle in the periparturient period. Journal of Dairy Science. 82:12821295.

Veech, R.L. (2004). The therapeutic implications of ketone bodies: The effects of ketone bodies in pathological conditions: ketosis, ketogenic diet, redox states, insulin resistance, and mitochondrial metabolism. Prostaglandins Leukot. Essential Fatty Acids. 70:309-319.

Wildman, E.E., Jones, G.M., Wagner, P.E., Bowman, R.L. (1982). A dairy cow body condition scoring system and its relationship to selected production characteristics. Journal of Dairy Science. 65: 495-501.

Zarrin, M., De Matteis, L., Vernay, M.C., Wellnitz, O., van Dorland, H.A., Bruckmaier, R.M. (2013). Long-term elevation of $\beta$-hydroxybutyrate in dairy cows through infusion: Effects on feed intake, milk production, and metabolism. Journal of Dairy Science. 96:2960 - 2972.

Zarrin, M., Grossen-Rosti, L., Bruckmaier, M.R., Gross, J.J. (2017). Elevation of blood Bhydroxybutirate concentration affects glucose metabolism in dairy cows before and after parturition. Jorunal of Dairy Science. 100: 2323 - 2333. 